\title{
Linx
}

Revue des linguistes de l'université Paris X Nanterre

$8 \mid 1996$

Du dire et du discours

\section{La langue brésilienne (des effets de la colonisation sur la langue)}

Eni Puccinelli-Orlandi

\section{(2) OpenEdition}

Journals

Édition électronique

URL : http://journals.openedition.org/linx/1139

DOI : 10.4000/linx.1139

ISSN : 2118-9692

Éditeur

Presses universitaires de Paris Nanterre

\section{Édition imprimée}

Date de publication : 1 septembre 1996

Pagination : 41-50

ISSN : 0246-8743

\section{Référence électronique}

Eni Puccinelli-Orlandi, « La langue brésilienne (des effets de la colonisation sur la langue) », Linx [En ligne], 8 | 1996, mis en ligne le 24 juillet 2012, consulté le 30 avril 2019. URL : http:// journals.openedition.org/linx/1139; DOI : 10.4000/linx.1139

Ce document a été généré automatiquement le 30 avril 2019.

Département de Sciences du langage, Université Paris Ouest 


\title{
La langue brésilienne (des effets de la colonisation sur la langue)
}

\author{
Eni Puccinelli-Orlandi
}

1 Il y a plusieurs sortes d'objets symboliques qui concourent à la formation d'un pays. En effet, c'est de la production de ces objets et du rapport qu'établissent les sujets avec ceuxci que résultent aussi bien les sens attribués à ce pays que les sens que prennent ces sujets en tant qu'ils se définissent comme des serfs ou des citoyens, c'est à dire, en tant qu'ils se définissent par rapport à la formation de "leur" pays.

Dans la perspective discursive, on le sait, sujet et sens se produisent en même temps. En effet, par le seul fait de produire du sens, le sujet se signifie à lui même. Aussi, nous pouvons dire que dans la formation de l'imaginaire social, l'histoire de la construction de la langue nationale est structurellement liée à la constitution de la forme historique du sujet sociopolitique qui se définit dans son rapport à la formation du Pays (ou de la Nation, ou de l'Etat).

3 Si l'on considère la périodisation de l'histoire brésilienne, il y a différents processus discursifs qui s'établissent selon les différentes conceptions de société. La période coloniale, le XVIIIe siècle (et la législation sur l'esclavage), ainsi que la pensée politique libérale positiviste du XIXe siècle au Brésil en sont des exemples marquants.

Dans cette étude on cherchera à expliciter les processus de signification par lesquels, dans la construction du Brésil, la langue nationale prend sens dans un contexte de cultures en contact, c'est à dire, l'européenne et l'amérindienne.

Dans ce cas, il est très important de constater que la construction imaginaire de l'unité et de l'homogénéité joue un rôle crucial dans l'établissement d'une identité, dans un pays spécifique, avec ses formes spécifiques de gouvernement et avec une langue (nationale). 


\section{Langue imaginaire et langue fluide}

6 En 1984, à partir de la notion d'"indien imaginaire" (G. Thérien, UQAM, Canada) ${ }^{1}$, et de mon expérience des cultures indigènes brésiliennes, j'ai établi la distinction entre langue imaginaire et langue fluide ${ }^{2}$.

7 La langue imaginaire est celle que les analystes fixent avec leurs systématisations et la langue fluide est celle qui ne se laisse pas fixer dans des systèmes et des formules. Théoriquement, cette distinction se fonde sur celle de Pêcheux et Gadet (1983) quand ils parlent du "corps plein du langage" et des "processus de construction des grammaires". Dans la pratique historique brésilienne je considère le "tupi jésuite" (Orlandi et Souza, 1988 et Orlandi, 1987) sous la rubrique de langue imaginaire. Dans un travail de 1987 "La Danse des Grammaires" j'ai proposé d'inclure sous la catégorisation de langue imaginaire la construction de la langue nationale, dans notre cas, le portugais standard.

La prise en compte du contact historique et culturel entre les langues, met en jeu nécessairement le rapport entre ces deux notions, la langue imaginaire et la langue fluide, dans ses limites mouvantes.

\section{Hétérogénéité Linguistique}

9 J. Authier (1982) établit la notion d'hétérogénéité énonciative en considérant que tout dire est constitué par la présence de l'Autre. Nous nous inspirons de cette définition en lui donnant toutefois d'autres spécifications. Aussi parlerons-nous d'hétérogénéité linguistique ${ }^{3}$ pour désigner, dans le domaine des pays de colonisation, une langue, comme le portugais ou l'espagnol en Amérique Latine, qui fonctionne selon le principe d'une identité que je qualifierai de double.

10 Selon la définition de la formation discursive (Pêcheux, 1975, Courtine, 1982) nous pouvons parler la même langue mais parler différent. Par ailleurs, nous pouvons en dire autant des langues de colonisation qui sont considérées comme les mêmes mais qui s'historicisent différemment dans leurs rapports à l'histoire de la formation du pays. C'est le cas du portugais du Brésil et celui du Portugal. Nous parlons la "même" langue mais nous parlons différent.

11 A côté de l'hétérogénéité dont parle J. Authier (ibid) en mettant en cause le rapport à l'autre et à l'Autre, et de la différence dont parle M. Pêcheux où interviennent différentes formations discursives dans la même langue, nous proposons de distinguer un autre aspect de l'hétérogène et de la différence. Nous appelons donc hétérogénéité linguistique le jeu qui fait qu'il y a dans notre langue un double fond par lequel le même abrite néanmoins un autre, un différent historique qui le constitue même s'il a l'apparence du même : le portugais brésilien et le portugais portugais se recouvrent comme s'ils étaient la même langue mais ils ne sont pas la même langue. Ils produisent des discours différents. Ils signifient différemment. Du point de vue discursif il est possible d'accéder à ce tour de prestidigitation par lequel dans le même "lieu" il y a néanmoins une double présence, au moins deux discours différents, effets d'un clivage de deux histoires dans le rapport avec la langue portugaise: celle du Portugal et celle du Brésil. Nous, les Brésiliens, quand nous parlons le portugais nous sommes toujours dans ce point de disjonction obligée. Notre langue signifie dans une filiation de mémoire hétérogène. Ces 
deux langues se relient à des interdiscursivités distinctes comme si elles étaient néanmoins les mêmes. Cet effet d'homogénéité est l'effet de l'histoire de la colonisation.

Les conséquences de ce fait sont innombrables. L'une d'entre elles, déjà évoquée par S. Gallo (1991) réfère au rapport écrit/oral au Brésil. Le rapport du portugais du Portugal avec l'écriture (d'abord le latin et ensuite le roman portugais) est déterminé historiquement de façon absolument différente de celle du portugais du Brésil (latin écrit, nhengatou et portugais écrit). Cette différence non visible dans l'histoire de la grammaire produira néanmoins des effets très importants dans la scolarité quand les professeurs voient leur projet d'enseignement de l'écriture déboucher sur l'échec : il y a une oralité (celle qui vient en ligne directe des langues de tradition orale comme le nhengatou) qui ne sera jamais légitimée et qui résistera à la scolarisation. Dans notre conception, cet échec est un des effets de l'hétérogénéité linguistique. La compréhension de cet effet fait appel à des raisons historiques dans la matérialité même de la langue.

13 Cette hétérogénéité est d'autant plus importante que son action est moins repérable, puisque les processus historiques ne sont pas directement visibles dans la langue. C'est ainsi que travaillent les objets symboliques dans leur historicité. Dans le cas du portugais, nous pouvons dire qu'il s'agit de systèmes symboliques divers (celui du Brésil et celui du Portugal) avec des histoires (linguistiques) différentes mais ayant en apparence la même matérialité empirique (matière brute, donné). De là résultent les équivoques. L'observation de la même matérialité empirique ne laisse pas voir (méconnait) la diversité de la matérialité historique. Dans la perspective discursive nous proposons de définir la langue par sa forme matérielle et non, comme chez les linguistes, comme forme abstraite ${ }^{4}$; et c'est par la réflexion sur la forme matérielle du portugais brésilien que l'on peut le mieux repérer les différences que nous essayons d'expliciter.

14 Ces formes de présence de l'altérité nous amènent à considérer que les rapports de confrontation entre les cultures ne se manifestent pas toujours comme des confrontations mais qu'ils peuvent se présenter aussi dans leur indistinction, autrement dit, on ne peut pas être toujours sûr de la partie qui nous est réservée dans les processus identitaires. En d'autres termes, l'identité n'est pas identique à elle-même, n'est pas toujours discernable dans sa spécificités. Cela dépend de son histoire constitutive. Et nous arrivons là à la question la plus importante dans ce travail : celle du fonctionnement de la mémoire dans la constitution de l'identité des langues.

\section{Mémoire et Polysémie}

15 Je reprends ici un fait que j'ai analysé (1992a) comme discours fondateur de la brésilienneté. Il s'agit de l'énoncé Em se plantando tudo dá (Si l'on plante tout pousse) formulé par Pero Vaz de Caminha (le responsable du journal de bord de Pedro Alvares Cabral, le "découvreur" du Brésil). Pour bien mener l'analyse de cet énoncé j'ai consulté le texte original et j'ai été surprise par la forme de l'énoncé que j'ai trouvé: Em tal maneira é graciosa que, querendo-a aproveitar, dar-se-á nela tudo, por causa das águas que tem (elle est si belle que si l'on veut en profiter, tout poussera, avec les eaux qu'elle a). C'est une formulation très éloignée de celle que l'on répète dans notre mémoire scolaire, ce qui m'a fait conclure que ce n'est pas l'énoncé dans sa forme empirique qui reste dans la mémoire mais son image énonciative, autrement dit, sa forme historique. Ce fait, par lui même, nous indique que, s'agissant du symbolique, la mémoire n'a pas à être fidèle, ni à suivre une ligne droite. Le rapport est indirect et médiatisé par des facteurs historico-textuels, 
c'est à dire, par des conditions de production de l'énoncé, des rapports de sens et de forces que le caractérisent. Ce sont des espaces de mémoire, discontinus. Ils se constituent dans des parcours, des réseaux de filiation historique qui donnent une forme (informent) aux interprétations. Ils font ainsi partie de notre imaginaire social de brésilien. Ils produisent "les choses à savoir" (M. Pêcheux, 1983), dans le fonctionnement historique du symbolique : celui qui délimite le dicible, le signifiable, dans le rapport avec l'interdiscours (mémoire du dire).

16 A l'indistinction possible, s'ajoute alors cette autre caractéristique de la mémoire, son caractère indirect qui ne travaille pas avec les empiries mais avec des images énonciatives, ces images attestant, à leur tour, le caractère discontinu de la mémoire symbolique.

17 Ce qui fonctionne donc dans notre mémoire linguistique, dans l'identité nationale, ce sont les images construites dans notre rapport à la langue portugaise, notre "version" brésilienne de ce rapport.

18 A ces faits vient s'en ajouter un autre : la polysémie et l'incomplétude; discontinue et aussi incomplète, la mémoire n'est pas fermée et achevée, mais dispersée et polysémique.

19 En effet, un même objet est affecté par deux mouvements de sens, voire plus. C'est ça la polysémie: la simultanéité de mouvements différents de sens dans le même objet symbolique. Il y a composition de sens dans la polysémie et la mémoire soutient cette composition, autrement dit, la mémoire est par nature polysémique.

20 Cette conception du mouvement de sens, de la polysémie comme composition, peut rendre compte des différences entre des lignes idéologiques dans l'affectation de différentes valeurs aux objets symboliques. De là la différence entre des tendances diverses comme le formalisme ou le sociologisme etc. Elles travaillent à l'intérieur de la distinction entre forme et contenu - en méconnaissant donc la notion de forme matérielle, c'est à dire l'inscription de l'histoire dans la forme linguistique - et elles fonctionnent dans une conception de la polysémie prise par l'illusion du contenu (Orlandi, 1992). Ainsi, elles effacent le mouvement du sens et se fixent sur des contenus qui sont des effets de l'historicité. Dans notre perspective nous ne séparons pas forme/ contenu. Nous travaillons la matérialité de l'objet symbolique. Et la définition de la polysémie que nous avons énoncée ci-dessus est de cette nature, autrement dit, c'est une définition discursive (matérielle) et non une définition de contenu.

21 Ces commentaires nous aideront à bien situer la question de la polysémie par rapport à notre objet de réflexion, la langue brésilienne. Si nous traitons cette question de façon discursive (matérielle) nous pouvons comprendre quelque chose de très important $d u$ point de vue de l'identité.

Quand nous avons fait référence, précédemment, au mode de fonctionnement du portugais-brésilien sous la forme de la disjonction obligée, nous visions l'indistinction mais nous étions en train de situer aussi la polysémie, comme nous venons de la définir. Si nous pensons le rapport du portugais-brésilien à la colonisation, il y a fonctionnements simultanés de sens, par des mouvements symboliques distincts. Cela ne veut pas dire que pour distinguer nos langues nous devons comparer empiriquement les sens dans leurs différences. Cela signifie plutôt qu'il y a une marque de distinction dans la matérialité historique de ces systèmes symboliques qui surdétermine, dans cette composition de sens, le portugais brésilien. Cela veut dire que le brésilien signifie différemment du portugais quand il signifie en portugais (brésilien). Voilà la duplicité constitutive, 
l'hétérogénéité, la polysémie qui est à la base même de l'exercice de la langue. Mieux encore: le portugais et le brésilien n'ont pas le même sens. Ce sont des langues matériellement différentes. Et cette différence travaille par son invisibilité (son indistinction). L'occurrence des différences visibles sont des "accidents" (accent, tours syntaxiques) empiriques, dépassables du point de vue de l'identité linguistique (langue imaginaire). Ce qui les distingue néanmoins profondément (langue fluide) ce sont les propriétés inscrites dans la différence de constitution de leurs processus de signification spécifiques inscrits dans leur matérialité linguistico-historique. Là, ce qui n'est pas attesté par l'observation empirique vaut néanmoins comme trait d'identité. Le rapport de colonisation est un "événement linguistique" (J.Guilhaumou, 1989) ainsi que la proclamation de l'indépendance ${ }^{6}$. Le rapport de colonisation produit ce clivage disjonction obligée, hétérogénéité linguistique, duplicité - qui atteint la matérialité de la langue brésilienne. C'est une marque de naissance travaillée de plusieurs façons tout au long de son histoire.

Cette histoire d'identité de la langue nationale se prolongera ainsi au travers de multiples événements, tels que les traités entre le Brésil et le Portugal, la fondation des Académies, les règlements scolaires et autres. C'est cette histoire que nous commençons à connaître. Et ce texte est un premier pas en direction de cette forme de connaissance qui est aussi une prise de position par rapport à l'histoire de la connaissance sur la langue et sur la constitution de la langue nationale au Brésil.

\section{Quelques conséquences fondamentales}

Il y a des conséquences théoriques et pratiques qui découlent de cette méthode discursive de penser la langue dans son histoire et dans son fonctionnement. Nous aimerions mettre l'accent sur deux d'entre elles.

En termes théoriques, cette méthode d'analyse qui consiste à penser la constitution de la langue nationale dans son historicité, par la prise en compte de sa forme matérielle, nous situe dans un domaine spécifique de l'histoire des sciences. Nous construisons ainsi une forme de connaissance sur la langue qui constitue, par elle même, un mode de rapport avec le langage et une position théorique définie : celle qui étudie la production des objets symboliques en mouvement, comme partie d'une histoire dans laquelle les sujets et les sens se construisent. Nous ne sommes pas dans le domaine d'une épistémologie positiviste mais historique (D. Lecourt, 1978) où il n'y a pas séparation entre sujet-objet, donné-fait, intérieur-extérieur, abstrait-concret et où l'histoire n'est pas conçue comme évolution et continuité, mais discontinuité et fonctionnement.

Dans le domaine de la pratique, il reste à observer que, dans le rapport enseignementapprentissage à l'école, on parle bel et bien de l'échec de l'enseignement de la langue (soit la grammaire, soit la lecture, soit l'écriture), échec constaté devant l'inconsistance de connaissances qui ne durent pas: l'étudiant sait dans l'immédiat mais peu après il "oublie". En réalité il n'y a pas eu apprentissage. Ce qui manque c'est l'inscription du sujet dans un processus historique; autrement dit, ce qui ne fait pas sens dans l'histoire du sujet n'adhère pas, ne se fixe pas. Plus spécialement, au lieu qu'il y ait inscription du sujet dans la filiation historique, inscription dans la mémoire de l'objet symbolique où le sujet se constitue, il y a eu effacement si bien qu'il n'y a pas de sens pour le sujet, cela ne fait pas sens dans son histoire. En conséquence c'est hors discours (et ici "hors de" ne signifie pas extérieur mais exclu, effacé, mis sous silence). 
Dans l'exemple que nous avons donné, ne pas prendre en compte les deux formes d'oralité qui découlent de deux histoires différentes fait que l'on essaie de passer, avec violence, d'une oralité qui fait partie de l'histoire de la langue brésilienne à une écriture dont une oralité correspond à une "autre" histoire. C'est une impossibilité. Et le résultat ce n'est pas, à mon avis, l'échec mais l'inconsistance historique, la résistance à ce qui ne fait pas sens. D'autre part, l'observation de ces aspects historiques peut faire mieux comprendre la nécessité de ces passages et aussi le moyen de les effectuer.

Dans l'histoire de la langue brésilienne on ignore un moment très important de sa constitution, celui où la langue parlée était la "langue générale" (le nhengatou) et la langue écrite était le latin, puis le portugais, le nhengatou n'ayant pas "mérité" la légitimation d'une écriture. Cette méconnaissance a eu pour conséquence fondamentale dans la culture brésilienne, l'oubli de son rapport à sa tradition orale. Cette tradition orale est néanmoins un trait de notre histoire culturelle, qui montre que les Brésiliens - à la différence des Européens - ont un rapport très faible à l'écriture et un attachement très fort à l'oral. Dans la communication administrative, par exemple, les gens lisent les papiers mais ne "croient" qu'à ce qui est dit oralement. Le droit aussi fonctionne beaucoup par appel à la jurisprudence (droit anglo-saxon) et non pas aux lois écrites (droit continental). Ce trait historique a sûrement son poids quand il s'agit du passage que doit faire l'élève, à l'école, de l'oral à l'écriture. Cependant, l'immédiateté des rapports scolaires, le poids de l'idéologie colonisatrice européenne (qui "naturalise" le rapport à l'écriture) et l'effacement des trajets historiques produits par la réduction de l'enseignement de la langue à la dominance de la perspective grammaticale (et non discursive), conduisent de préférence l'étudiant non pas à un passage mais à une impasse. De là la résistance qui reçoit le nom d'“échec scolaire".

Ces considérations d'ordre pédagogique sont à peine une ébauche rapide des implications de la question historico-discursive qui se présente dans la conception même de langue nationale ${ }^{7}$. Il y a d'autres implications de nature différente tout aussi dignes de réflexion.

Il n'y a donc pas à méconnaître l'histoire du sujet et de la langue dans la production de la connaissance du sujet sur la langue.

\section{BIBLIOGRAPHIE}

AUTHIER, J. (1982) Hétérogénéités énonciatives, Langages, Ref. Paris.

BATALlA, B. (1990) Mexico Profundo (La civilisacion negada), Grijalbo, Mexico.

COURTINE, J.-J. (1982) Définition d'orientations théoriques et construction de procédures en analyse de discours, Philosophiques, Paris, 2, vol. IX.

GALLO, S. (1992) Discurso da Escrita e Ensiño, Unicamp, Campinas.

GUILHAUMOU, J. (1989) La Langue Politique et la Révolution Française, Paris, Méridiens, Klincksieck.

LECOURT, D. (1978) Pour une critique de l'Épistémologie, Maspero, Paris. 
ORLANDI, E. (1985) A língua imaginária e a língua fluida : o português brasileiro sob a influência das linguas indigenas, Colóquio, IEL.

ORLANDI, E. e de SOUZA, T.C.C. (1988) A língua imaginária e a língua fluida : dois métodos de trabalho com a linguagem, in Política Linguística na América Latina, Pontes, Campinas.

ORLANDI, E. (1990) La Danse des Grammaires, Cóloquio de Nice, 1987 e versão modificada em Terra à Vista, Cortez/Unicamp, São Paulo.

ORLANDI, E. (1993) Vão Surgindo sentidos, in O Discurso Fundador, Pontes, Campinas.

PÊCHEUX, M. (1975) Les Vérités de La Palice, Maspero, Paris.

PÊCHEUX, M. et GADET, F. (1983) La langue introuvable, Paris, Maspero.

PÊCHEUX, M. (1992) Discours : Structure ou Événement?, in L'inquiétude du Discours, D. Maldidier, Ed. des Cendres, Paris.

\section{NOTES}

1. Très récemment j'ai pris connaissance, au Mexique, d'un livre qui étudie la confrontation des cultures et qui s'appelle Mexico Profundo, de B. Batalla. Il distingue aussi dans ce cas le pays imaginaire du pays profond et il montre que la notion même d'indien est une notion imaginaire produite par la nécessité européenne dans son rapport aux découvertes.

2. Colloque (des jeudis) présenté au Département de Linguistique, IEL, Unicamp, 1985.

3. Le déplacement que je propose ici repose sur le fait que J. Authier parle de l'énonciation et nous parlons de la langue même, autrement dit de sa constitution historique. De là résulte la notion, dans notre étude, d'hétérogénéité linguistique. Il y a dans ce cas un travail de la langue sur la langue (non pas métalangage mais disjonction (historique)).

4. Il faut rappeler ici que la distinction que je propose entre forme abstraite et forme matérielle accentue l'importance du rapport établi par l'analyse de discours entre langue et histoire. Par la forme matérielle nous pouvons travailler l'inscription de l'historique dans la langue, l'investissement du sens dans la syntaxe (ou la grammaire), de façon à suivre le principe selon lequel la matérialité de l'idéologie est le discours et la matérialité spécifique du discours est la langue. Nous établissons ainsi la relation entre la forme-sujet et la forme du sens par la notion de matérialité discursive : linguistique et historique.

5. Une blague racontée par les Brésiliens est ici très utile: un chauffeur portugais avait, à Lisbonne, deux passagers brésiliens dans son taxi. Les Brésiliens bavardaient joyeusement. À un moment donné, le chauffeur se tourne vers les Brésiliens et leur dit : "quelle langue parlez-vous pour que, moi, je comprenne tout ?" Le même et le différent, la familiarité et l'éloignement, jouent ici le jeu de l'indistinction dans l'histoire et dans la mémoire.

6. Pour cet aspect, je me réfère aux travaux présentés, en 1991, par notre équipe, à Paris VII, dans le Colloque "La citoyenneté au Brésil et en France" (sous presse).

7. Dans un projet dont je suis responsable au Brésil et qui est coordonné par S. Auroux en France nous développons des études et recherches sur l'Histoire des Idées Linguistiques au Brésil et sur la Constitution de la Langue Nationale. Ce projet s'inscrit dans un projet plus large d'analyse de discours que j'anime dans le Département de Linguistique, sous le nom de "Discours, Signification, Brésilienneté". 


\section{RÉSUMÉS}

On montre dans cet article comment la construction d'une langue nationale est liée à la construction du sujet sociopolitique. L'exemple est celui de la langue brésilienne, constituée à travers différents processus discursifs. La distinction entre langue imaginaire et langue fluide permet de rendre compte de l'hétérogénéité linguistique liée à l'histoire, que représente le cas des portugais brésilien.

This article shows how the construction of a national language is connected to the construction of the sociopolitical subject. We take as an example the Bresilian language, constructed through different discursive processes. The distinction between "langue imaginaire" and "langue fluide" permits us to explain the linguistic heterogeneity tied to the history, illustrated by the case of Bresilian language.

\section{AUTEUR}

\section{ENI PUCCINELLI-ORLANDI}

